\title{
Design of coaxial aircell fixture for the measurement of electromagnetic properties
}

\author{
V. Seetha Rama Raju \\ Physics Research Lab, CVR College of Engineering (Autonomous), Hyderabad, Telangana 501510, India \\ E-mail address: vengalarajuseetharamaraju@gmail.com
}

\begin{abstract}
Coaxial aircells are designed and fabricated to measure the electromagnetic properties of ferrite materials in the frequency range from $1 \mathrm{MHz}$ to $3.6 \mathrm{GHz}$. These $\mathrm{S}$ parameters are actually measured connecting the aircell to a vector network analyzer (VNA). The electromagnetic properties such as complex permittivity and complex permeability are extracted using Nicolson-Ross-Weir (NRW) method and also suitable air-gap corrections are made. To optimize the measured result and to estimate the error, the aircells are characterized in terms of their phase constant and resistivity of the aircell conductor. The measurements clearly showed that the electrical length is longer than the mechanical length of the aircell at all frequencies. The arithmetic mean of the resistivity of aircell which is of $7 \mathrm{~mm}$ line size and $60 \mathrm{~mm}$ length is about $66 \mathrm{n} \Omega \mathrm{m}$. This paper presents a simple method by which the phase constant and resistivity of the aircell can be determined accurately. This is done with the transmission measurements made using a VNA.
\end{abstract}

Index Terms - Coaxial aircell, S-parameters, Complex permittivity, Complex permeability.

\section{INTRODUCTION}

The measurement of complex permeability $\left(\mu^{*}=\mu^{\prime}-i \mu^{\prime \prime}\right)$ and complex permittivity $\left(\varepsilon^{*}=\varepsilon^{\prime}-i \varepsilon^{\prime \prime}\right)$ of materials at high-frequency is used in microwave communication devices, electromagnetic interference (EMI) shielding, electromagnetic compatibility (EMC), field sensors, microwave heating and magnetic recording etc.

A number of methods have been developed for measuring permittivity and permeability. These techniques include free-space methods [1-3], waveguide techniques [4, 5], open-ended coaxialprobe techniques [6, 7], cavity resonators [8], and dielectric-resonator techniques [9]. Each method has its range of applicability and its inherent limitations. For instance, however cavity based techniques are precise, yet not broadband, are typically restricted to low-loss materials. The waveguide methods do not experience the radiation loss like free-space methods and at the same time give accurate results. However, each waveguide operates at the limited frequency band. Coaxial line method gives accurate results and allows measurement in a wider frequency range [10-12].

Coaxial air dielectric transmission lines can be very useful as references, or standards, for high-precision impedance measurement at RF and microwave frequencies [13-15]. These aircells do not contain dielectric support that are usually used to hold the center conductor of the aircell coaxially 
inside the outer conductor. The absence of dielectric support medium, along with the use of highconducting materials for the aircell's conductors, air as the dielectric and high precision connectors at both ends of the line, means that such lines exhibit near ideal properties, and exhibit very low insertion loss.

A measurement utilizing the transmission / reflection (TR) technique continues by setting a specimen in an area of coaxial aircell and measuring the two-port complex S-parameters, ideally by a vector network analyzer (VNA). The scattering equations relate the measured scattering parameters to the permittivity and permeability of the material. However, for these aircells to be used for such applications, they must be characterized accurately in terms of their propagation characteristics and impedance. This includes the understanding of a small amount of loss in the aircell and the associated impact of this loss on other characteristics. A simple to-execute test technique has been exhibited in this paper for the determination of the resistivity of the aircells conductor including VNA estimations of the S-parameters of the aircell.

\section{EXPERIMENTAL METHOD}

\section{A. Sample holder}

A coaxial aircell consists of a hollow cylindrical transmission line. The inner walls have a smooth contour and acts as the sample holder. Both outer ends are threaded to secure the connectors to both test ports on a Network Analyzer. A center conductor runs through the center of the hollow cylinder and provides the connection, and is secured in place by the threaded cables on both the sides.

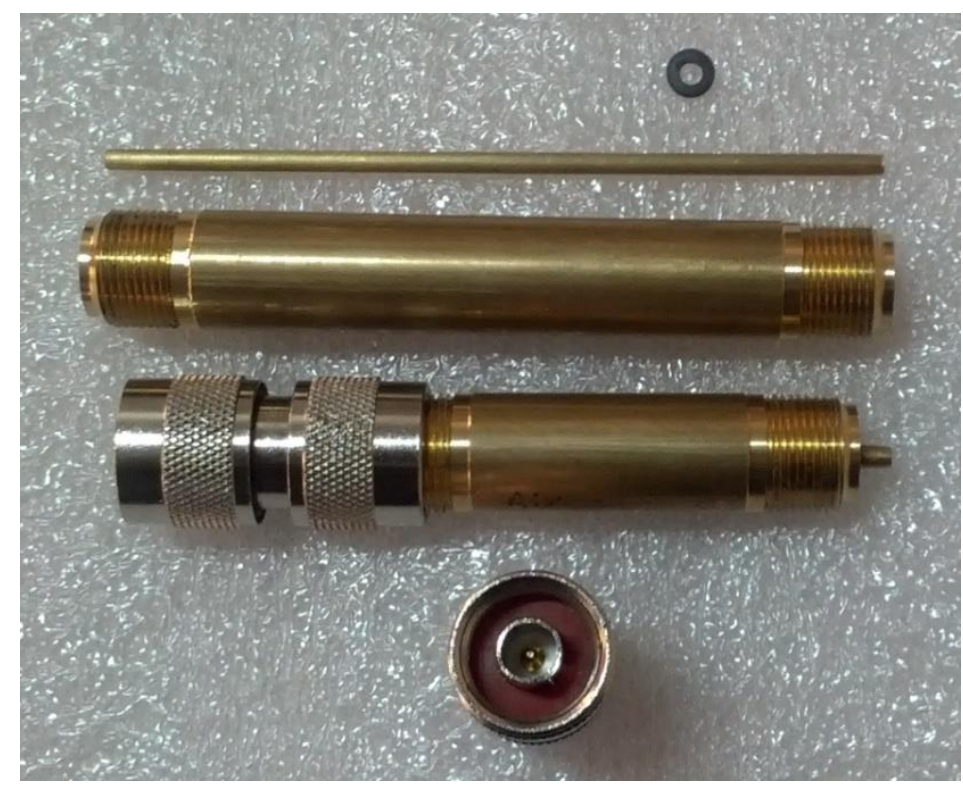

Fig. 1(a). Photograph of the coaxial aircell.

Figures 1 (a) and 1 (b) show the photograph and the structure of the coaxial aircell sample holder fabricated in this study. The sample holder is machined on Jobber XL CNC turning centre in our 
college workshop with machine position accuracy of $6 \mu \mathrm{m}$. The sample holder and the VNA are connected though male to male $\mathrm{N}$ type connectors with standard $7 \mathrm{~mm}$ coaxial cables. The cell is made of Brass and its dimensions are adapted to match the precise $\mathrm{N}$ connectors used. All these components had a $50 \mathrm{ohm}$ characteristic impedance to prevent mismatch.

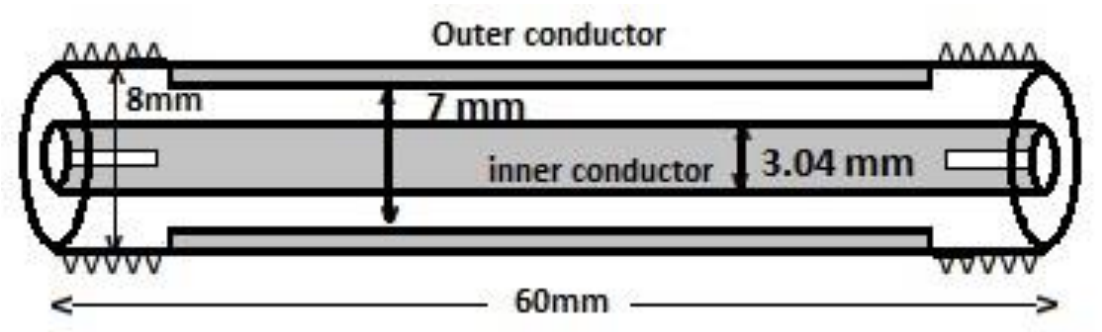

Fig. 1(b). Structure of the coaxial aircell.

\section{B. Measurement setup}

In this work measurement of S-parameters are made using Rohde \& Schwarz ZVH 4 Cable and Antenna analyzer / Network analyzer. Full two-port calibration was initially performed on the test setup in order to remove errors due to the directivity, source match, load match, isolation and frequency response in both the forward and reverse measurements. The R\&S ZVH analyzer uses ZVH 29 standard kit for short, open, load and through calibration at each port. The calibration also establishes the reference planes for the measurement test ports. Before we proceed for the measurement of the ferrite samples, the fabricated coaxial aircells without samples are characterized for phase constant and resistivity.

\section{Test samples}

The ferrite samples $\left(\mathrm{Mg}_{0.2} \mathrm{Cu}_{0.3} \mathrm{Zn}_{0.5} \mathrm{Fe}_{2} \mathrm{O}_{4}, \mathrm{Ni}_{0.53} \mathrm{Cu}_{0.12} \mathrm{Zn}_{0.35} \mathrm{Fe}_{2} \mathrm{O}_{4}\right.$ and $\left.\mathrm{Co}_{0.4} \mathrm{Zn}_{0.6} \mathrm{Fe}_{2} \mathrm{O}_{4}\right)$ are finally pressed into the toroidal-shaped samples of $7.00 \mathrm{~mm}$ outer diameter and $3.04 \mathrm{~mm}$ inner diameter with the height of $4.0 \mathrm{~mm}$. The scattering parameters of the toroidal samples that correspond to the reflection (S11 and S22) and transmission (S21 and S12) are measured by the vector network analyzer using a coaxial transmission / reflection method in the frequency range of $1 \mathrm{MHz}$ to 3.6 GHz. The toroids tightly fit into the coaxial measurement cell.

\section{Phase constant and Resistivity measurement}

The aircell of length $\mathrm{L}$ connected between the two ports of reference plane will have a phase change of the signal to travel between two ports. The electrical length of the line may therefore be calculated from the measured phase using the equation

$$
\mathrm{L}=\frac{(2 \pi n-\theta)}{\beta}
$$

where $\beta$ is called phase constant, $\theta$ is the phase (in radians) measured by the VNA and $n$ is an integer $(n=0,1,2, \ldots)$ used to take account of the number of complete wavelengths that the length of aircell contains at a given frequency. The value of ' $n$ ' is determined from knowledge of the length of the coaxial aircell. The determined electrical length of the aircell (from (1)) compared with its 
physical length (obtained from a precision mechanical measurement). If the two length determinations (mechanical and electrical) agree to within the expected uncertainty, then the VNA phase measurement is verified as being of an acceptable accuracy (again, to within the expected uncertainty of measurement).

In practice, the aircells are not lossless, they exhibit a small amount of loss owing to the finite conductivity (or non-zero resistivity) of the metals used to fabricate the aircell's conductors. The attenuation constant $\alpha$ is related to a transmission coefficient (say S21) of a length L, of perfectly matched line (i.e. $|\mathrm{S} 11|=|\mathrm{S} 22|=0$ ), by

$$
\alpha=\frac{-\log _{e}|S 21|}{L}
$$

the resistivity $\rho$ of a line is related to $\alpha$ as follows [16]:

$$
\rho=\left[\frac{200 \alpha \mathrm{b}}{1+(\mathrm{b} / \mathrm{a})}\right]^{2} \frac{\pi}{\mu_{0} \mathrm{f}}
$$

where $\mathrm{a}$ and $\mathrm{b}$ are the radii of the aircell centre and outer conductors respectively

From equations (2) and (3), it is clear that, at any given frequency, $\rho$ can be determined from the measurement of S21.

\section{E. Extraction of electromagnetic properties from S-parameters}

The complex permeability $\left(\mu^{*}\right)$ and permittivity $\left(\varepsilon^{*}\right)$ of the ferrite samples are determined from the scattering parameters using the NRW models using the following equations $[17,18]$.

$$
\mu^{*}=\frac{1+\Gamma}{\Lambda(1-\Gamma) \sqrt{\frac{1}{\lambda_{0}^{2}-\frac{1}{\lambda_{c}^{2}}}}}
$$

Where $\Gamma$ is reflection coefficient, $\lambda_{0}$ is the free space wavelength and $\lambda_{c}$ is the cutoff wavelength and

$$
\frac{1}{\Lambda^{2}}=-\left(\frac{1}{2 \pi \mathrm{L}} \ln \left(\frac{1}{\mathrm{~T}}\right)\right)^{2}
$$

The permittivity can be defined as

$$
\varepsilon^{*}=\frac{\lambda_{0}^{2}}{\mu_{\mathrm{r}}}\left(\frac{1}{\lambda_{\mathrm{c}}^{2}}-\left[\frac{1}{2 \pi \mathrm{L}} \ln \left(\frac{1}{\mathrm{~T}}\right)\right]^{2}\right)
$$

Equation (12) and (13) have an infinite number of roots since the imaginary part of the term $\ln \left(\frac{1}{\mathrm{~T}}\right)$ is equal to $\mathrm{i}(\theta+2 \pi n)$ where $\mathrm{n}=0, \pm 1, \pm 2 \ldots$, the integer of $\left(\frac{L}{\lambda_{g}}\right)$. The ' $\mathrm{n}$ ' can be determined by finding $\lambda_{g}$ wavelength in sample.

\section{F. Airgap correction:}

Air-gaps are created if the machined sample does not fit the sample holder's cross-section and make close contact with the cavity walls [19]. As with the parallel-plate capacitor, ideal experimental accuracies of $5 \%$ can be achieved, but air-gaps and fixture problems can decrease the accuracy to $15 \%$ [20]. This is a $10 \%$ increase in experimental uncertainty. Larger air-gaps will inevitably increase the 
uncertainty. There are two types of air-gap correction; coaxial and waveguide gap correction. The respective correction is applied once the permittivity data has been obtained after data conversion.

Coaxial gap correction calculation can be determined by first describing the dimension of the sample and coaxial holder into three terms [21]:

$$
\begin{aligned}
& L_{1}=\ln \frac{D_{2}}{D_{1}}+\ln \frac{D_{4}}{D_{3}} \\
& L_{2}=\ln \frac{D_{3}}{D_{2}} \\
& L_{3}=\ln \frac{D_{4}}{D_{1}}
\end{aligned}
$$

Where $D_{1}$ is the diameter of the inner conductor in the aircell, $D_{2}$ is the inner diameter of the toroidal sample, $D_{3}$ is the outer diameter of the sample, and $D_{4}$ is the diameter of the coaxial aircell itself (see Figure 2).

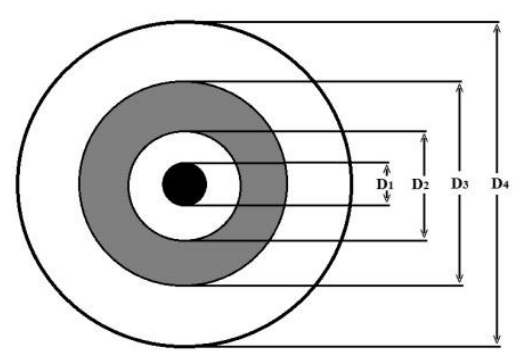

Fig. 2. Coaxial sample in holder with air gaps.

With these terms, the corrected real and imaginary part of the permittivity can be determined as

$$
\begin{gathered}
\varepsilon_{c}^{\prime}=\varepsilon_{m}^{\prime} \frac{L_{2}}{L_{3}-L_{1} \varepsilon_{m}^{\prime}} \\
\varepsilon_{c}^{\prime \prime}=\left(\varepsilon_{c}^{\prime} \times \frac{\varepsilon_{m}^{\prime \prime}}{\varepsilon_{m}^{\prime}}\right) \frac{L_{3}}{L_{3}-L_{1} \varepsilon_{m}^{\prime}\left(1+\left[\frac{\varepsilon_{m}^{\prime \prime}}{\varepsilon_{m}^{\prime}}\right]^{2}\right)}
\end{gathered}
$$

Where $\varepsilon_{c}=$ corrected permittivity values and $\varepsilon_{m}=$ measured permittivity values

With the same terms, the corrected real and imaginary part of the permeability is described as

$$
\begin{aligned}
& \mu_{c}^{\prime}=\mu_{m}^{\prime} \frac{L_{3}-L_{1}}{L_{2}} \\
& \mu_{c}^{\prime \prime}=\mu_{m}^{\prime \prime} \frac{L_{3}}{L_{2}}
\end{aligned}
$$

Where $\mu_{c}=$ corrected permeability values and $\mu_{m}=$ measured permeability values

\section{RESULT AND DISCUSSION}

The difference between the mechanical length and electrical length of the coaxial aircell is shown in figure 3. It is clear from the figure that, if the line is assumed to be lossless, the electrical length is longer than the mechanical length of the aircell at all frequencies. The electrical length also varies as a function of frequency (owing to the dispersion in the aircell not being taken into account). However, when the loss is taken into account, the electrical length of the aircell shows better agreement with the known mechanical length. Figure 4 therefore indicates that it is necessary to know 
the resistivity of the aircell before it can be used as a reference artefact for verifying VNA phase measurements.

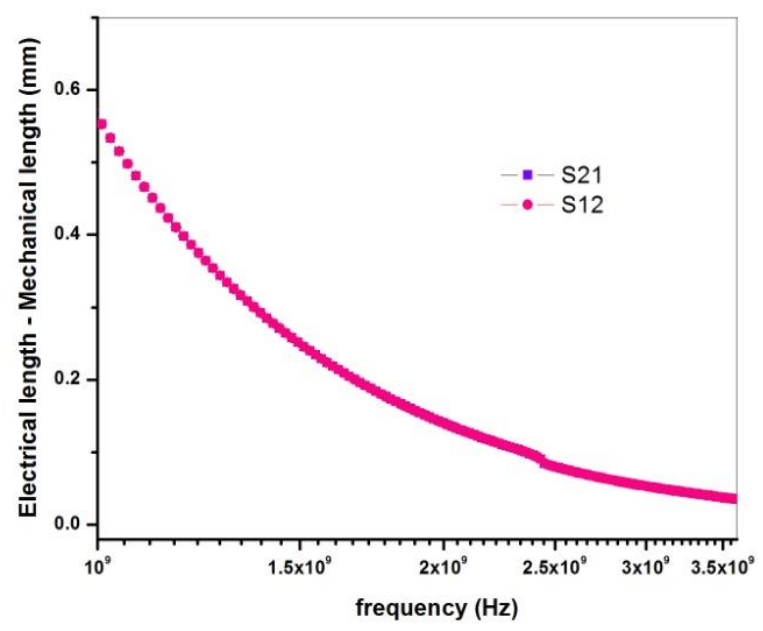

Fig. 3. Difference between mechanical and electrical length of the coaxial aircell.

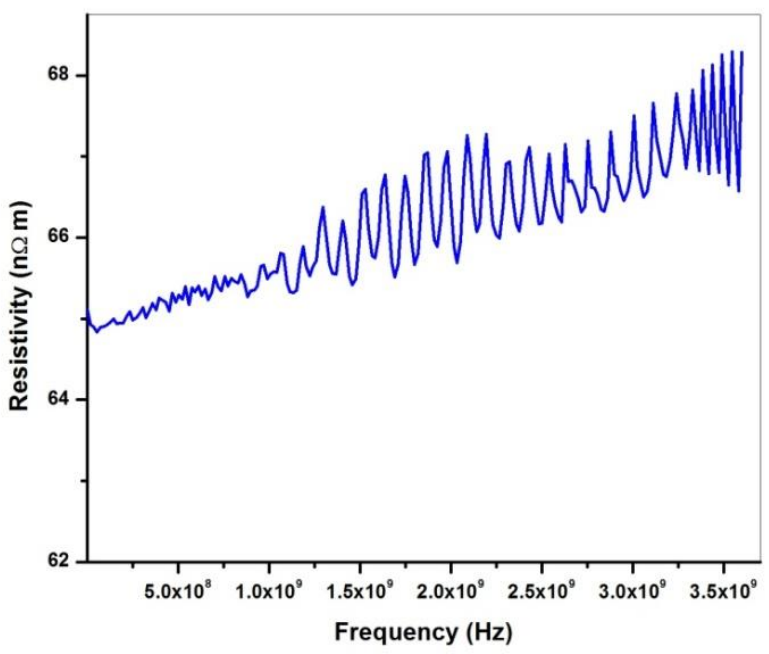

Fig. 4. Resistivity versus frequency of the aircell conductor.

Figure 4 shows the resistivity determinations for these lines plotted as a function of frequency. The arithmetic mean of the resistivity of aircell of $7 \mathrm{~mm}$ line size of length of $60 \mathrm{~mm}$ is about $66 \mathrm{n} \Omega \mathrm{m}$. These results show good agreement with the earlier determinations of resistivity [16], and so this provides some degree of validation for the technique presented in this paper. Any deviation in the values of resistivity depends on the composition of the aircell conductor.

The complex permeability spectra of the ferrite samples obtained from NRW method is shown in figure 5 . The value of the real part of permeability $\mu^{\prime}$ for present ferrites is more than 500 in the frequency range of $1 \mathrm{MHz}$ to $50 \mathrm{MHz}$. Then the values of permeability are found to decrease with an increase in frequency up to $180 \mathrm{MHz}$. Finally, frequency dispersion occurred at 200 to $500 \mathrm{MHz}$. 
The loss component $\mu^{\prime \prime}$ rises to a pronounced peak as $\mu^{\prime}$ falls. This is the resonance type of permeability dispersion. The resonance/relaxation frequency $f_{\mathrm{r}}$, at which $\mu^{\prime \prime}$, has a maximum value, is above $200 \mathrm{MHz}$ for all ferrites.
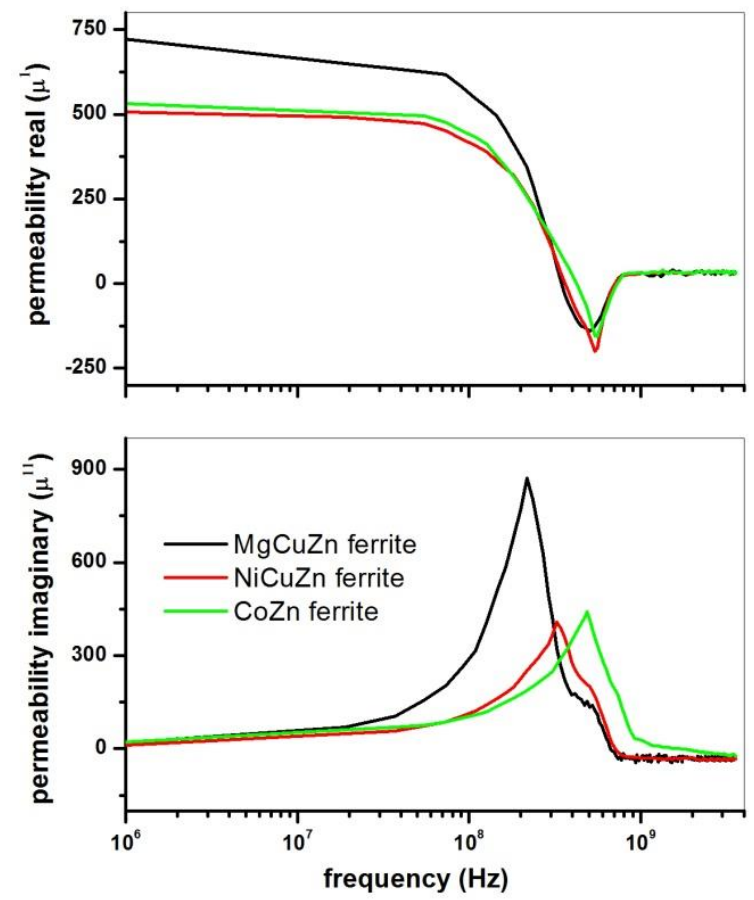

Fig. 5. Complex permeability spectra of ferrites.

It is known that the complex permeability is composed of two different magnetization mechanisms and those are the spin rotational magnetization and the domain wall motion. The first one is a relaxation type factor and its dispersion is inversely proportional to frequency. Otherwise, the domain wall contribution is of resonance type and depends on the square of the frequency [22]. Usually, there is a relevant interplay between the resonance due to the domain wall motion and relaxation due to the spin rotation. Domain wall resonance is usually observed at lower frequency while the spin rotational relaxation is observed in $\mathrm{MHz}$ frequency range.

The high frequency permeability of spinel ferrite is governed by Snoek's law as $\left(\mu_{s}-1\right) f_{r}=$ $\gamma / 3 \pi M_{s}$ where, $\mu_{s}$ is the static permeability, $f_{r}$ is the resonance frequency, $M_{s}$ is the saturation magnetization and $\gamma$ is the gyromagnetic ratio [23]. It follows from the equation that a change in $\mu_{\mathrm{s}}$ due to variations in material microstructure is followed by an opposite change in $\mathrm{f}_{\mathrm{r}}$.

The complex permittivity spectra of the ferrite samples obtained from NRW method is shown in figure 6. The complex permittivity of any material, in general, is due to dipolar, electronic, ionic, and interfacial polarizations. At low frequencies, dipolar and interfacial polarizations are known to play the most important role [24]. Moreover, the polarization in ferrites is through a mechanism similar to the conduction process [25]. By electron exchange between $\mathrm{Fe}^{2+} \leftrightarrow \mathrm{Fe}^{3+}$, one obtains local 
displacement of electrons in the directions of the electric field and these electrons determine the polarization.

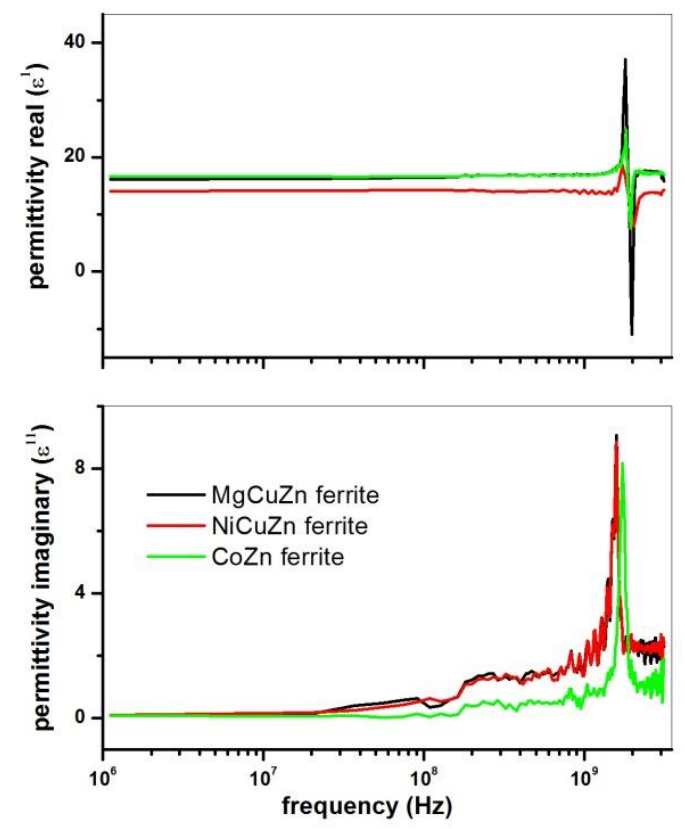

Fig. 6. Complex permittivity spectra of ferrites.

In ferrites, it is well known that the samples consist of well-conducting grains separated by poorly conducting grain boundaries. The electrons reach the grain boundary through hopping and if the resistance of the grain boundary is high enough, electrons pile up at the grain boundaries and produce polarization. Koops explained this by considering the heterogeneous structure of polycrystalline ferrites as equivalent to a series parallel combination of resistors and capacitors [26]. However, as the frequency of the applied field increases, the electrons reverse their direction of motion more often. This decreases the probability of electrons reaching the grain boundary and as a result the polarization decreases.

Therefore, the dielectric constant decreases with increasing frequency of the applied field. Ultimately, as the field frequency increases, the dipoles will barely have started to move before the field reverses, and try to move the other way. At this stage, the field is producing virtually no polarization of the dielectric. This process is generally called relaxation and the frequency beyond which the polarization no longer follows the field. Relaxation arises from the inertia of the system of charges and restoring force acting on the charges which oppose the force due to the applied electric field. Thus, these systems combine inertia and restoring force, whereby resonance is possible rather than relaxation.

The dielectric losses in ferrites are generally reflected in the resistivity, materials with low resistivity exhibiting high dielectric loss and vice versa. The frequency variation of imaginary part of 
permeability can be explained on the basis of Koop's model [26]. The resonance peak around $1 \mathrm{GHz}$ can be explained as when the hopping frequency of electrons is equal to the applied field frequency, maximum electrical energy is transferred to the oscillating ions and power loss shoots up, thereby resulting in resonance. The measured results of complex permeability and complex permittivity in this work are in good agreement with the published reports measured with impedance analyzers [27-29].

\section{Conclusions}

A new coaxial aircell sample holder has been designed and fabricated to measure the Sparameters in transmission / reflection methods. The aircell is characterized for phase constant and resistivity for optimization of result using a simple method discussed in this paper. The complex permeability and complex permittivity of the ferrite materials are extracted from measured Sparameters in the frequency range of $1 \mathrm{MHz}$ to $3.6 \mathrm{GHz}$ using NRW method.

\section{Acknowledgements}

The author is very much thankful to Dr. K. S. Nayanatara for making VNA available for measurements. I would like to thank M. Srinivas Reddy, Y. Srinivas Goud and K. Venkat Reddy for their help in machining the aircell. I also would like to thank Dr. C. V. Raghava (Chairman, CVR College of engineering) and Dr. C. Madhusudana Reddy (Advisor) for their encouragement.

\section{References}

[1] D. K. Ghodgaonkar V. V. Varadan, V. K. Varadan, "A free-space method for measurement of dielectric constants and loss tangents at microwave frequencies," IEEE Trans. Instrum. Meas., vol. 38, pp. 789-793, 1989.

[2] P. Skocik, P. Neumann, "Measurement of Complex Permittivity in Free Space," Procedia Eng., vol. 100, pp. 100-104, 2015.

[3] F. C. Smith, B. Chambers, and J. C. Benett, "Methodology for accurate free-space characterization of radar absorbing materials," Proc. Inst. Elect. Eng. Sci. Meas. Technol., vol. 141, pp. 538-546, 1994. [4] M. J. Akhtar, L. Feher, M. Thumm, "Measurement of dielectric constant and loss tangent of epoxy resins using a waveguide approach,” in Proc. IEEE Antennas Propag. Soc. Int. Symp., vol. 1, pp. $3179-3182,2006$.

[5] H. Soleimania, Z. Abbasb, N. Yahyaa, H. Soleimanib, M. Yeganeh Ghotbic, "Determination of complex permittivity and permeability of lanthanum iron garnet filled PVDF-polymer composite using rectangular waveguide and Nicholson-Ross-Weir (NRW) method at X-band frequencies," Measurement., vol. 45, pp. 1621-1625, 2012.

[6] B. Filali, F. Boone, J. Rhazi, G. Ballivy, "Design and Calibration of a Large Open-Ended Coaxial Probe for the Measurement of the Dielectric Properties of Concrete," IEEE Trans. Microw. Theory Tech., vol. 56, pp. 2322-2328, 2008. 
[7] D. M. Hagl, D. Popovic, S. C. Hagness, J. H. Booske, M. Okoniewski, "Sensing Volume of Open-Ended Coaxial Probes for Dielectric Characterization of Breast Tissue at Microwave Frequencies,” IEEE Trans. Microw. Theory Tech., vol. 51, pp. 1194-1206, 2003.

[8] J. Sheen, "Microwave Measurements of Dielectric Properties Using a Closed Cylindrical Cavity Dielectric Resonator,” IEEE Trans. Dielectr. Electr. Insul., vol. 14, pp. 1139-1144, 2007.

[9] J. Krupka, A. P. Gregory, O. C. Rochard, R. N. Clarke, B. Riddle, J. B. Jarvis, "Uncertainty of complex permittivity measurements by split-post dielectric resonator technique,” J. Eur. Ceram. Soc., vol. 21, pp. 2673-2676, 2001.

[10] V. Shemelin, N. Valles, "Improved accuracy of measurements of complex permittivity and permeability using transmission lines,” Nucl. Instr. Meth. Phys. Res. A., vol. 767, pp. 385-396, 2014.

[11] J. B. Jarvis, R. Geyer, P. Domich, "Improvements in transmission line permittivity and permeability measurements," in Precision Electromagnetic Measurements (CPEM) Conf. on Jun 1990, pp. 232-233.

[12] J. Xu, M. Y. Koledintseva, Y. Zhang, Y. He, B. Matlin, R. E. DuBroff, J. L. Drewniak, J. Zhang, "Complex Permittivity and Permeability Measurements and Finite-Difference Time-Domain Simulation of Ferrite Materials," IEEE Trans. Electromagn. Compat., vol. 52, pp. 878-887, 2010.

[13] B. O. Weinschel, "Air-filled coaxial lines as absolute impedance standards,” Microw. J., vol. 7, pp. 47-50, 1964.

[14] I. A. Harris, R. E. Spinney, "The realization of high-frequency impedance standards using air spaced coaxial lines,” IEEE Trans. Instrum. Meas., vol. 13, pp. 265-272, 1964.

[15] K. H. Wong, "Using precision coaxial air dielectric transmission lines as calibration and verification standards,” Microw. J., vol. 31, pp. 83-92, 1998.

[16] G. J. Kilby, N. M. Ridler, "Comparison of theoretical and measured values for attenuation of precision coaxial lines,” Electron. Lett., vol. 28, pp. 1992-1994, 1992.

[17] A. M. Nicolson and G. F. Ross, "Measurement of the intrinsic properties of materials by timedomain techniques,” IEEE Trans. Instrum. Meas. vol. 19, pp. 377-382, 1970.

[18] W. B. Weir, "Automatic measurement of complex dielectric constant and permeability at microwave frequencies," Proc. IEEE., vol. 62, pp. 33-36, 1974.

[19] J. Baker-Jarvis, C. Jones, B. Riddle, M. Janezic, R. Geyer, J. Grosvenor and C. Weil, "Dielectric and magnetic measurements: A survey of nondestructive, quasinondestructive, and process- control techniques," Research in Nondestructive Evaluation, vol. 7, pp. 117-136, 1995.

[20] J. Krupka, A. Abramowicz, "Measurements of Electromagnetic Properties of Materials at Microwave Frequencies," XXXII International Conference of IMAPS - CPMT IEEE Poland Pułtusk 21 - 24 September 2008.

[21] J. Baker-Jarvis, "Transmission/Refection and Short-Circuit Line Permittivity Measurements," NIST Technical Note 1341, July 1990. 
[22] C.A. Stergioun, V. Zaspalis, "Analysis of the complex permeability of NiCuZn ferrites up to 1 GHz with regard to $\mathrm{Cu}$ content and sintering temperature," Ceram. Int., vol. 40, pp, 357-366, 2014.

[23] T. Nakamura, "Snoek's limit in high-frequency permeability of polycrystalline Ni-Zn, Mg-Zn, and Ni-Zn-Cu spinel ferrites,” J. Appl. Phys., vol. 88, pp. 348-354, 2000.

[24] S. Zaima, T. Furuta, and Y. Yasuda, "Conduction mechanism of leakage current in $\mathrm{Ta}_{2} \mathrm{O}_{5}$ films on Si prepared by LPCVD,” J. Electrochem. Soc., vol. 137, pp. 2876-2882, 1990.

[25] V. Seetha Rama Raju, "Effect of $\mathrm{Ta}_{2} \mathrm{O}_{5}$ addition on the electrical and magnetic properties of nanocrystalline MgCuZn ferrites,” J. Mater. Res., vol. 29, pp. 2220-2228, 2014.

[26] C. G. Koops, "On the Dispersion of Resistivity and Dielectric Constant of Some Semiconductors at Audio frequencies,” Phys. Rev. Lett., vol. 83, pp. 121-124, 1951.

[27] V. Seetha Rama Raju, "Complex permeability spectra of PbO and Ta2O5 added nanocrystalline MgCuZn ferrites,” J. Magn. Magn. Mater., vol. 382, pp. 84-87, 2015.

[28] T. Krishnaveni, B. Rajini Kanth, V. Seetha Rama Raju, S. R. Murthy, "Fabrication of multilayer chip inductors using Ni-Cu-Zn ferrites,” J. Alloys Compd., vol. 414, pp. 282-286, 2006.

[29] T. Slatineanua, A. R. Iordana, V. Oanceaa, M. N. Palamarua, I. Dumitrub, C. P. Constantinb, O. F. Caltun, "Magnetic and dielectric properties of Co-Zn ferrite," Mater. Sci. Eng. B., vol. 178, pp. 1040-1047, 2013. 